Trinity University

Digital Commons@ Trinity

Chemistry Faculty Research

Chemistry Department

9-1-2010

\title{
Circular Dichroism Spectroscopy in the Undergraduate Curriculum
}

Adam R. Urbach

Trinity University, aurbach@trinity.edu

Follow this and additional works at: https://digitalcommons.trinity.edu/chem_faculty

Part of the Chemistry Commons

\section{Repository Citation}

Urbach, A. R. (2010). Circular dichroism spectroscopy in the undergraduate curriculum. Journal of Chemical Education, 87(9), 891-893. doi: 10.1021/ed1005954

This Post-Print is brought to you for free and open access by the Chemistry Department at Digital Commons @ Trinity. It has been accepted for inclusion in Chemistry Faculty Research by an authorized administrator of Digital Commons @ Trinity. For more information, please contact jcostanz@trinity.edu. 


\title{
Circular Dichroism Spectroscopy in the Undergraduate
}

\section{Curriculum}

\author{
Adam R. Urbach \\ Department of Chemistry, Trinity University, San Antonio, TX 78212 \\ adam.urbach@trinity.edu
}

Adam R. Urbach is Associate Professor of Chemistry at Trinity University, specializing in bioorganic chemistry. His group applies circular dichroism spectroscopy to studies on the molecular recognition of proteins and nucleic acids by designed molecules.

Circular dichroism spectropolarimetry (CD) is a method of optical spectroscopy that seems in most practical ways like UV-visible spectroscopy. The main difference between the two methods is that $\mathrm{CD}$, instead of measuring the absorbance of light as a function of wavelength, measures the difference in absorbance of left versus right circularly polarized light as a function of wavelength. A CD spectrum is an observation of the structure of a chiral compound; it often serves as a "fingerprint" of the structure of biological molecules such as proteins and nucleic acids. For this reason, $\mathrm{CD}$ has been broadly applied in biochemistry and in many other areas ranging from organometallic to nanoscale chemistry. Despite its exceptional ease of use and connection to central topics in chemistry, however, $\mathrm{CD}$ remains rare in the undergraduate curriculum. This column 
provides a brief introduction to the theory and practice of CD spectroscopy and a discussion of its advantages in the undergraduate curriculum and the required instrumentation. For a more complete treatment of $\mathrm{CD}$, the reader is referred to excellent comprehensive references (1,2) including an early series in this Journal (3).

\section{Introduction to $\mathrm{CD}$}

Most students have worn polarized sunglasses, prescription eyeglasses, or even 3D movie glasses. For this reason, they are likely to be familiar with the concept of planepolarized light, in which the electric field vector oscillates in a single plane. Few students, however, are familiar with circularly polarized (CP) light, in which the electric field vector rotates about the axis of light propagation in a helical path. For example, in right $\mathrm{CP}$ light the field rotates in a clockwise direction when the light is traveling away from the viewer, just as the fingers on your right hand curl clockwise when your right thumb points away from you. CP light can be thought of as "chiral light", in which left and right CP light are analogous to a pair of enantiomers. The physical and chemical properties of enantiomers are identical in every way except that they interact differently with other asymmetric species. Similarly, left and right CP light have identical properties except that they interact differently with asymmetric molecules.

Plane-polarized light comprises equal and opposite components of left and right $\mathrm{CP}$ light, like a racemic mixture of enantiomers. A CD spectropolarimeter (or CD spectrometer) separates plane-polarized light into the two components and then measures the difference in absorbance of each component by a sample $\left(\Delta \mathrm{A}=\mathrm{A}_{\mathrm{L}}-\mathrm{A}_{\mathrm{R}}\right)$ as a function of wavelength. Positive CD signal therefore indicates a greater absorbance of 
left versus right $\mathrm{CP}$ light. Using Beer's law, $\Delta \mathrm{A}=\Delta \varepsilon C l$, where $\Delta \varepsilon$ is the differential molar absorptivity $\left(\mathrm{M}^{-1} \mathrm{~cm}^{-1}\right)$ of left versus right (i.e., $\left.\varepsilon_{\mathrm{L}}-\varepsilon_{\mathrm{R}}\right) \mathrm{CP}$ light, $C$ is the molar concentration, and $l$ is the path length in $\mathrm{cm}$. The quantity $\Delta \varepsilon$ is known as the molar circular dichroism and, analogous to molar absorptivity, is a wavelength-dependent property intrinsic to a given substance under a given set of environmental conditions (e.g., temperature, $\mathrm{pH}$, and so on). The $\mathrm{CD}$ spectrum is a plot of molar ellipticity, [ $\theta]$, versus wavelength, where $[\theta]=3298.2 \Delta \varepsilon$.

A molecule that exhibits CD must contain a chromophore in an asymmetric environment. Each chromophore has a characteristic absorptivity in one or more regions of the UV-visible spectrum. If the chromophore is in an asymmetric electronic environment, it may also preferentially absorb left or right $\mathrm{CP}$ light. The resulting CD spectrum will therefore appear within the absorbance spectral range of the molecule. The CD spectrum is the sum of the contributions of each chromophore; in this way, the spectrum can be used as a "fingerprint" to identify that compound or as a metric for monitoring changes in structure.

\section{Common Uses of CD.}

CD has been applied in many areas of chemistry, but by far the most common application is the study of biomolecular structure. The structures of proteins and nucleic acids are ideal for CD studies because each residue has a chromophoric component in an asymmetric environment, and the secondary and tertiary structures impart unique CD spectral characteristics. The far-UV region $(<250 \mathrm{~nm})$ contains information about secondary structure such as $\alpha$-helix, $\beta$-sheet, or random-coil (Figure 1 ). The relative 
contributions of $\alpha$-helix and $\beta$-sheet to the CD signal of a globular protein can therefore be viewed qualitatively, or one can deconstruct a spectrum quantitatively (4). The nearUV region $(<250 \mathrm{~nm})$ contains less predictable information about tertiary structure from chromophoric groups on phenylalanine, tyrosine, tryptophan, and cysteine. The visible region shows information on ligand-metal interactions in metalloproteins.

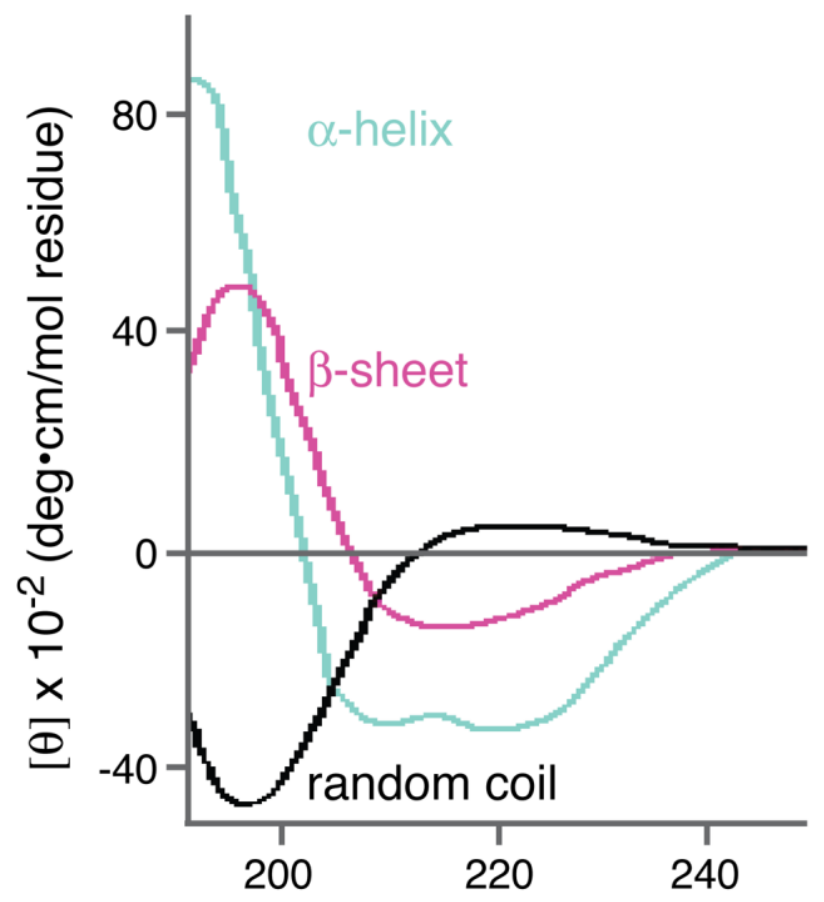

Figure 1. Illustration of $\mathrm{CD}$ spectra that represent proteins with predominantly $\alpha$-helix, $\beta$-sheet, or random coil structure.

In addition to its use in characterizing molecular structure, CD spectroscopy is particularly powerful for observing changes in structure. One of the most common applications of $\mathrm{CD}$ involves monitoring the change in the structure of a protein or nucleic acid upon heating. The increase in temperature causes a transition from the natural, folded state to an unfolded state, and the $\mathrm{CD}$ spectrum changes accordingly. The 
temperature at the center of this transition (i.e., the melting temperature) is a benchmark indicator of the stability of a protein or nucleic acid: the more stable the fold, the higher the temperature required to unfold it. This feature translates to practice as a convenient method for measuring the stability of a biomolecule by simply performing a temperature scan. For this reason, a Peltier temperature controller is typically purchased along with a CD spectrometer.

\section{CD in the Classroom.}

Numerous protocols have been published on the use of CD spectroscopy in the classroom, including several from this Journal (5-11). Some aim to introduce CD to students as an unusual method for measuring common chemical parameters (e.g., pKa) (6), but most utilize the unique features of $\mathrm{CD}$ as a spectral polarimetry technique that enables confirmation of the spectral fingerprint of protein structure $(7,10)$, measurement of the stability (11) or heat capacity (9) of a protein, determination of absolute configuration $(5,8)$, or monitoring the synthetic conversion of one chiral organic compound into another (8). These examples highlight the simple yet powerful way in which CD spectroscopy can be used to demonstrate basic concepts, such as molecular asymmetry, as well as advanced concepts, such as the thermodynamic parameters that govern protein folding. These advantages make $\mathrm{CD}$ an attractive topic for both classroom and laboratory curricula.

Conceptually, $\mathrm{CD}$ offers a relatively simple context in which to relate structure to spectroscopy. In sophomore organic chemistry, students learn about classical polarimetry, in which a chiral molecule rotates plane-polarized light to an extent that depends on the 
specific rotation, $\alpha$. Seldom discussed, however, is the photophysical connection between chirality and the ability to rotate plane-polarized light (i.e., the intrinsic optical birefringence of a chiral sample), and students are thus left with a vague connection of molecular chirality to a numeric value of $\alpha$. By contrast, the analogy of CP light to an enantiomer, as described above, seems more intuitive, and the results of a CD experiment are captured graphically in a spectrum, providing more for students to observe and discuss. For example, in the overlaid spectra of D- vs. L-tryptophan (Figure 2) it is immediately clear that the two enantiomers have an equal and opposite CD response and that $\mathrm{CD}$ is a wavelength-dependent phenomenon, analogous to absorptivity.

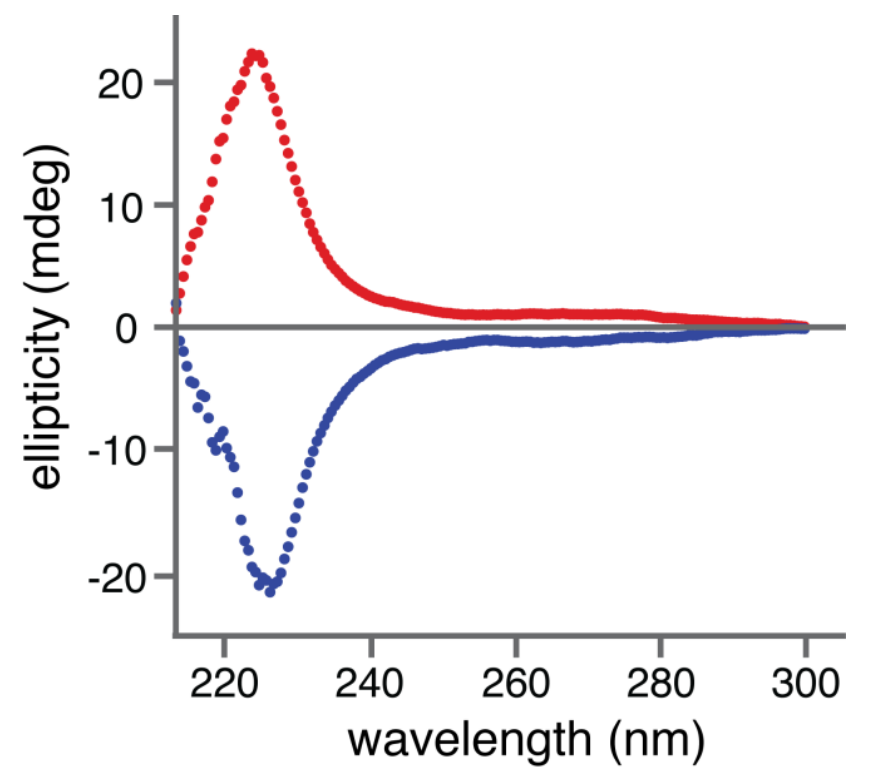

Figure 2. CD spectra of $1 \mathrm{mM}$ solutions of L-tryptophan (red) and D-tryptophan (blue) in $10 \mathrm{mM}$ sodium phosphate, $\mathrm{pH}$ 7.0, showing the symmetry in the spectra of these enantiomers.

In the teaching laboratory, $\mathrm{CD}$ spectroscopy is attractive for many of the same reasons as UV-visible spectroscopy. From a practical standpoint, the two techniques are 
very similar. For example, a student prepares a dilute sample of aqueous L-tryptophan, dispenses it into a standard $1 \mathrm{~cm}$ quartz cuvette, inserts the cuvette in the spectrometer, enters the desired UV-visible wavelength range, and acquires a spectrum over that range in a matter of minutes. If the student has already used a UV-visible spectrometer, then using a CD spectrometer will seem very familiar. The resulting spectrum has an appearance similar to a UV-visible spectrum, except that the peaks in a CD spectrum can be positive or negative. Just as a UV-visible spectrum is a graphic representation of color, with each chromophore displaying a unique spectrum, a CD spectrum is a graphic representation of molecular chirality, with each chiral compound showing a unique profile. From an instrumental analysis standpoint, the CD spectrometer is a simple extension of a single-beam UV-vis. Both use a broadband source, monochromator, and detector (photomultiplier-based for CD and most UV-vis). The main functional difference is that the CD uses a plane-polarizer and a photoelastic modulator (PEM), which separates the components of left and right CP light.

\section{Instrumentation Needs.}

A representative spectrometer is shown in Figure 3. Required instrumentation includes the spectrometer and computer $(\$ 70-80 \mathrm{k})$ and a streaming nitrogen source to keep oxygen out of the system. Most users will purchase a temperature controller to monitor changes in structure upon heating. I strongly recommend a Peltier-type controller (\$7$8 \mathrm{k}$ ), which has fine control and a fast response time. This controller requires a water bath and pump to circulate the water. We use a 5-gallon bucket with a simple aquarium pump for this purpose. The facility will require about six feet of bench, including four feet for the spectrometer, with nearby space for the nitrogen source. We use a liquid nitrogen tank 
because long temperature scans tend to deplete standard gas cylinders too quickly.

Therefore, costs of gas and demurrage need to be considered. Jasco, Inc. has the majority share of the United States market of CD spectrometers and auxiliary equipment (12). Instruments can also be purchased from Applied Photophysics; their Web site is an excellent source of information on the theory and application of CD (13).

\section{Conclusions}

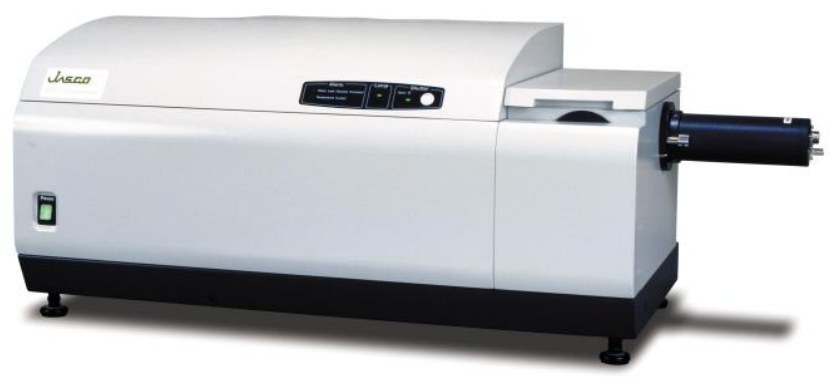

Figure 3. A representative CD spectrometer. The cylinder at right is the detector.

We acquired a CD spectrometer in 2007 through the Major Research Instrumentation program of the National Science Foundation. Although we use the instrument primarily for research, we have increased its presence in our laboratory courses significantly over the past two years. It takes a few hours per week and a few weeks per year to provide a hands-on experience to juniors and seniors. However, in the many cases where acquisition of an instrument is cost-prohibitive, I recommend introducing the theory of $\mathrm{CD}$ spectroscopy and the analysis of $\mathrm{CD}$ data into the classroom curriculum, particularly in biochemistry courses where its relevance is obvious. A recently constructed database for assimilating reference $\mathrm{CD}$ spectra of proteins provides a useful source of spectra for 
the classroom (14), and programs are freely available for deconvoluting protein CD spectra into the relative contributions from secondary structural elements (15).

Given the initial setup cost, CD spectroscopy may be too expensive for purely instructional purposes. Therefore, until lower-grade, less-expensive instruments become available, the accessibility of $\mathrm{CD}$ to the laboratory curriculum will likely be limited to institutions with active biochemical research programs and faculty who value the instructional benefits sufficiently to allow access to the instrument for the teaching laboratory.

Acknowledgement. We gratefully acknowledge the National Science Foundation (BIO-0718766) for an MRI grant that supported acquisition of our circular dichroism spectropolarimeter.

\section{Literature Cited}

1. Circular Dichroism: Principles and Applications, $2^{\text {nd }}$ Ed.; Nakanishi, K., Berova, N., Woody, R. W., Eds.; Wiley-VCH: 2000.

2. Rodger, A.; Nordén, B. Circular Dichroism and Linear Dichroism; Oxford University Press: 1997.

3. (a) Wong, K.-P. J. Chem. Educ. 1974, 51, A573-578; (b) Wong, K.-P. J. Chem. Educ. 1975, 52, A9-16; (c) Wong, K.-P. J. Chem. Educ. 1975, 52, A83-88.

4. (a) Whitmore, L.; Wallace, B. A. Nucl. Acids Res. 2004, 32, W668-673; (b) Whitmore, L.; Wallace, B. A. Biopolymers 2008, 89, 392-400.

5. Diaz-de-Villegas, M. D.; Urriolabeitia, E. P. J. Chem. Educ. 1999, 76, 77-78.

6. Cavaleiro, A. M. V.; Pedrosa de Jesus, J. D. J. Chem. Educ. 2000, 77, 1218-1220.

7. Bondesen, B. A.; Schuh, M. D. J. Chem. Educ. 2001, 78, 1244-1247.

8. Reed, N. A.; Rapp, R. D.; Hamann, C. S.; Artz, P. G. J. Chem. Educ. 2005, 82, 1053-1054.

9. Jones, C. L.; Bailey, C.; Bheemarti, K. K. J. Chem. Educ. 2006, 83, 1067-1070.

10. Russo, S.; Gentile, L. J. Chem. Educ. 2006, 83, 1850-1852.

11. Mehl, A. F.; Crawford, M. A.; Zhang, L. J. Chem. Educ. 2009, 86, 600-602.

12. Jasco Inc. circular dichroism page, http://www.jascoinc.com/Products/Spectroscopy/I-815-Circular-DichroismSpectrometer.aspx (accessed May 2010). 
13. Applied Photophysics Ltd. Circular dichroism page, http://www.photophysics.com/circulardichroism.php (accessed May 2010).

14. Protein Circular Dichroism Data Bank, http://pcddb.cryst.bbk.ac.uk/home.php (accessed May 2010).

15. Dichroweb, http://dichroweb.cryst.bbk.ac.uk/html/home.shtml (accessed May 2010). 